Copyright by the American Physical Society. Shneidman, V. A.; Zia, R. K. P., "Wulff shapes and the critical nucleus for a triangular Ising lattice," Phys. Rev. B 63, 085410 DOI: http://dx.doi.org/10.1103/PhysRevB.63.085410

PHYSICAL REVIEW B, VOLUME 63, 085410

\title{
Wulff shapes and the critical nucleus for a triangular Ising lattice
}

\author{
V. A. Shneidman \\ Department of Physics, New Jersey Institute of Technology, Newark, New Jersey 07102 \\ R. K. P. Zia \\ Department of Physics, Virginia Polytechnic Institute and State University, Blacksburg, Virginia 24061-0435
}

(Received 5 July 2000; published 5 February 2001)

\begin{abstract}
Equilibrium Wulff shapes and interfacial energies of two-dimensional "crystals" on a triangular lattice are considered. Asymptotic approximations are constructed for both the shapes and energies in the limit $T \rightarrow 0$ where crystals are close to perfect hexagons, and the limit $T \rightarrow T_{c}$ (critical temperature) where crystals have near-circular shapes. The intermediate temperature region is studied numerically, and accurate interpolating approximations are proposed. The relevance of the study to the nucleation problem is discussed.
\end{abstract}

DOI: 10.1103/PhysRevB.63.085410 PACS number(s): 68.65.-k, 64.60.Cn, 05.50.+q, 64.60.Qb

\section{INTRODUCTION}

In 1901 Wulff $^{1}$ showed how to construct a minimumenergy surface (" $S$ surface") that encloses a given volume $V$ of a crystal, provided one knows $\sigma(\vec{n})$, the anisotropic interfacial tension as a function of $\vec{n}$, the normal to the interface. This approach formed the basis of the modern understanding of real-life shapes of crystallites, with the variety of possibilities introduced by facets, corners, etc. ${ }^{2-4}$

Further interest in the Wulff construction came from twodimensional Ising systems. While some three-dimensional features (e.g., facets) are absent here for all positive temperatures $T$, the possibility of an exact evaluation of temperaturedependent anisotropic $\sigma(\vec{n})$ made such systems exceptionally interesting for analysis. Remarkably, there also exist experimental situations for which a two-dimensional understanding is of crucial importance. One can mention twodimensional islands on otherwise flat faces of threedimensional crystals (see, e.g., Refs. 5 and 6 and references therein), absorbed monolayers of surfactants, ${ }^{7}$ etc.

Applications of rigorous results, however, are often hindered by the fact that the Wulff construction provides a general prescription how to build an $S$ surface which may still require an appreciable effort before a practical implementation. Not surprisingly, alternative parametric representations of the Wulff construction were later developed for two dimensions $^{8}$ and for higher dimensions 9,10 in a coordinateinvariant way. A realization of such approaches for Ising systems on square or honeycomb lattices can be found, respectively, in Refs. 11 and 12. Nevertheless, even in the parametric representation results are usually given in terms of implicit dependencies, and further work is required both for an explicit determination of a Wulff shape and for an evaluation of the interfacial energies.

In this paper we extend the results of Ref. 12 for the case of a triangular lattice, obtaining the $S$ surface parametrically. We also asymptotically examine the limits $T \rightarrow T_{c}$ and $T$ $\rightarrow 0$ for both Wulff shapes and interfacial energies. Further, we construct elementary interpolating approximations for the entire temperature region; those are accurate, at worst, to one part in $10^{3}$. Such approximations can be useful for experiments $^{5,6}$ with the triangular lattice formed by atoms on the (111) plane of $\mathrm{Cu}$, as described in Sec. VII.

Another potential application of the results is related to nucleation from a metastable phase. It is expected that the work required to form a nucleus - the key parameter of the nucleation problem — can be obtained from the same equilibrium Wulff construction. ${ }^{13}$ Once this parameter is evaluated, available semiphenomenological expressions for the nucleation rates can be specified and tested against large-scale Monte Carlo simulations and numerical studies of transfer matrices. For a square lattice such comparisons were performed earlier. ${ }^{14-17}$ There are no comparative studies for a triangular lattice. Monte Carlo simulations of nucleation on such a lattice were carried out recently. ${ }^{18}$ Many of the kinetic aspects of the classical picture ${ }^{19-21}$ (e.g., the relation between nucleation and growth, transient nucleation effects ${ }^{22}$, etc.) are accurately reproduced in such simulations. However, the unavailability of an explicit simple expression for the surface energy of a nucleus prevented a crucial verification of the thermodynamics of the classical approach. In Sec. VI we evaluate the nucleation parameters, and in Sec. VII give some preliminary results of comparison.

\section{BACKGROUND}

The anisotropic interfacial tension can be obtained either directly (see, e.g., Ref. 23) or from its relation to the correlation length $\xi$ on a dual lattice: ${ }^{24}$

$$
\sigma(\vec{n})=T / \xi(\vec{u})
$$

Here $T$ is the temperature (Boltzmann constant is taken as unity), and $\vec{u}$ is a unit vector normal to $\vec{n}$. In turn, the correlation length can be extracted from the asymptote of the two-spin correlation function. ${ }^{12}$

For lattices with hexagonal symmetry, the duality ("startriangle" relation ${ }^{25}$ ) implies that the original reduced bond energy

$$
K=J^{\prime} / T
$$

is related to the one on a dual lattice by 


$$
\tanh K^{*}=\exp (-2 K)
$$

with the triangular lattice being dual to the honeycomb one, and vice versa.

The two-spin correlation function is known exactly for a triangular lattice. ${ }^{26}$ With this, the honeycomb interfacial tension can be evaluated, and the Wulff construction realized in a parametric form. ${ }^{12}$ In polar coordinates $(r, \theta)$ the result can be expressed implicitly as

$$
\begin{gathered}
\cosh \left\{\frac{r}{T} \sin \theta\right\}+\cosh \left\{\frac{r}{T} \sin \left(\theta+\frac{\pi}{3}\right)\right\} \\
+\cosh \left\{\frac{r}{T} \sin \left(\theta-\frac{\pi}{3}\right)\right\}=A,
\end{gathered}
$$

with

$$
A=A(K)=\left\{\left(\cosh 2 K^{*}\right)^{3}+\left(\sinh 2 K^{*}\right)^{3}\right\} / \sinh 2 K^{*} .
$$

Remarkably, if one evaluates the area $W(T)$ enclosed by the curves $r(\theta)$ in Eq. (4) three important physical characteristics - the polar radius $R(\theta)$ of an equilibrium surface of a crystal with volume $V$, the interfacial tensions in extremal directions, and the total interfacial energy-can be evaluated. The equilibrium surface is given by

$$
R(\theta)=r(\theta) \sqrt{V / W}
$$

i.e., just the scaled solution of Eq. (4). This solution has the dimension of $\sigma$, and for $\theta_{l}=l \pi / 6, l=0,1, \ldots, 11$ the interfacial tension in the direction normal to the surface is indeed given by $r\left(\theta_{l}\right)$. The total interfacial free energy of a crystal, $G$, is proportional to $\sqrt{V W}$, with a dimension-dependent proportionality constant. ${ }^{10}$ For the two-dimensional case considered, one obtains $G=2(V W)^{1 / 2}$.

As mentioned, the main goal of this paper is twofold. First we wish to adjust Eq. (4) for the case of a triangular lattice (which will lead to a different value of $A$ ), and to calculate the resulting interfacial energy of an equilibrium crystal. This will also determine the nucleus in a metastable case. Second, we intend to obtain asymptotic approximations for a Wulff shape in the limits $T \rightarrow 0$ and $T \rightarrow T_{c}$, which are valid for both triangular and honeycomb lattices. Understanding asymptotic limits will allow us to construct accurate interpolating approximations at intermediate temperatures.

Naively, one could think that once the interfacial tension is available for one type of hexagonal lattice, the tension for the other type is obvious from the duality relations. However, duality links the subcritical region of one lattice, where the interface exists, to the overcritical region of the other with no phase separation. Thus a preliminary transformation (the so-called $\Delta-Y$ transformation ${ }^{27}$ ) which links two $s u b$ critical lattices is required-see Sec. IV. First, however, we will introduce a few relations which are applicable for both lattices.

\section{SOME GENERAL EXPRESSIONS}

Using duality relation (3), one can express $A(K)$ as

$$
A(K)=\frac{1}{2 \sinh ^{2} K}+2 \sinh ^{2} K+1 .
$$

The value of $K$ here can be either the reduced bond energy of the original honeycomb lattice, as in Eq. (2), or it can correspond to bond energies of another lattice obtained by decorating a triangular lattice-see next Sec. IV. In both cases the expressions for $\sigma$ in the two principal directions are given by

$$
\begin{gathered}
\sigma_{1} \equiv r(0)=T \frac{2}{\sqrt{3}} \operatorname{arccosh}\left(\frac{A-1}{2}\right), \\
\sigma_{2} \equiv r(\pi / 2)=2 T \operatorname{arccosh}\left\{\sqrt{\frac{2 A+3}{4}}-\frac{1}{2}\right\} .
\end{gathered}
$$

For $T \rightarrow T_{c}$ the asymptote of $A(K)$ is given by

$$
A-3 \sim 4\left(\sinh ^{2} K-1 / 2\right)^{2} \text {. }
$$

The values of $\sigma_{1}$ and $\sigma_{2}$ rapidly converge to each other and to the value of

$$
\sigma_{c}(T) \sim T_{c} \frac{4}{\sqrt{3}}\left(\sinh ^{2} K-1 / 2\right) .
$$

This goes to zero as $T_{c}-T$, with the slope specified for each type of lattice by the temperature dependence of $K$.

One can also construct a more accurate (though somewhat less elementary) approximation for the interfacial tension using the fact that anisotropy appears only in higher-order terms in $T_{c}-T$. If $r$ is isotropic in Eq. (4), this equation can be averaged with respect to $\theta$, giving

$$
I_{0}[r / T]=A / 3,
$$

with $I_{0}$ being the modified Bessel function. Since an isotropic $r$ coincides with $\sigma$, the latter is given by

$$
\sigma \approx T I_{0}^{-1}[A / 3]
$$

In contrast to Eq. (10), where the error is quadratic in $T_{c}$ $-T$, the above expression is accurate up to $\left(T_{c}-T\right)^{5}$, at which point the anisotropic terms become important, as will be described in Sec. V A.

\section{IV. $\Delta-Y$ TRANSFORMATION FOR A TRIANGULAR LATTICE}

Consider a triangular lattice with a reduced bond energy

$$
L=J / T \text {. }
$$

A convenient parameter is

$$
x=\tanh (L),
$$

with criticality achieved at

$$
x_{c}=2-\sqrt{3}, \quad L_{c}=0.27465307 \ldots
$$


[note that this value is linked to the value of $K_{c}$ $=\operatorname{arctanh}(1 / \sqrt{3})$ by the duality relation].

"Decoration" of a triangular lattice consists of placing an additional spin in the center of each triangle (" $\Delta$ "), and connecting it with the surrounding three spins with new bonds $K$, giving " $Y$ ', patterns which form a honeycomb lattice. $^{25}$ (The $\Delta-Y$ transformation was briefly mentioned by Onsager, and further examined by Wannier ${ }^{28}$ ). The requirement of equivalence of partition functions on both lattices leads to the relation

$$
\tanh ^{2} K=\frac{x}{1+x+x^{2}}
$$

There are certain subtle questions as to what exactly happens to the interface upon this transformation in a general case, ${ }^{12}$ but no difficulties are expected for isotropic ferromagnetic bonds considered in the present work.

The new honeycomb lattice is subcritical, i.e., $K>K_{c}$, if the original triangular lattice is below $T_{c}$. From Eqs. (6) and (15), one obtains

$$
A(L)=\frac{2 x}{(1-x)^{2}}+\frac{(1-x)^{2}}{2 x}+1 .
$$

This relation should be used for $A$ in Eq. (4) in order to describe the triangular lattice. The expanded path to the result (of which the present work is only a small part) can be summarized as follows: One starts with a low-temperature (subcritical) triangular lattice with bonds $L$; the $\Delta-Y$ transformation gives a low-temperature honeycomb lattice with bonds $K$; the duality relation links this lattice to a hightemperature triangular lattice with bonds $K^{*}$. Finally, the known correlation function for the latter lattice allows one to evaluate the anisotropic interfacial tension and to realize the Wulff construction.

\section{WULFF SHAPES AND ENERGIES}

The scaled Wulff shapes $r(\theta) / J$ obtained from Eqs. (4) and (16) at various temperatures are shown in Fig. 1. At $T$ $=0$ the shape is a perfect hexagon which rather quickly changes to an almost ideal circle upon the increase of temperature; analytical and numerical reasons for the rapid disappearance of anisotropy will become apparent shortly.

Due to symmetry, the area can be calculated as

$$
W=6 \int_{0}^{\pi / 6} r(\theta)^{2} d \theta .
$$

To characterize $W$ we will introduce an 'effective', tension

$$
\sigma_{e f f}=\sqrt{W / \pi},
$$

which coincides with the actual interfacial tension in the isotropic high-temperature region. Otherwise, $\sigma_{\text {eff }}$ corresponds to an equivalent surface tension of a circular droplet with area and energy identical to those of an anisotropic crystal. The values of $\sigma_{e f f}$ are shown in Fig. 2 by solid lines together with anisotropic interfacial tensions given by Eqs. (7) and

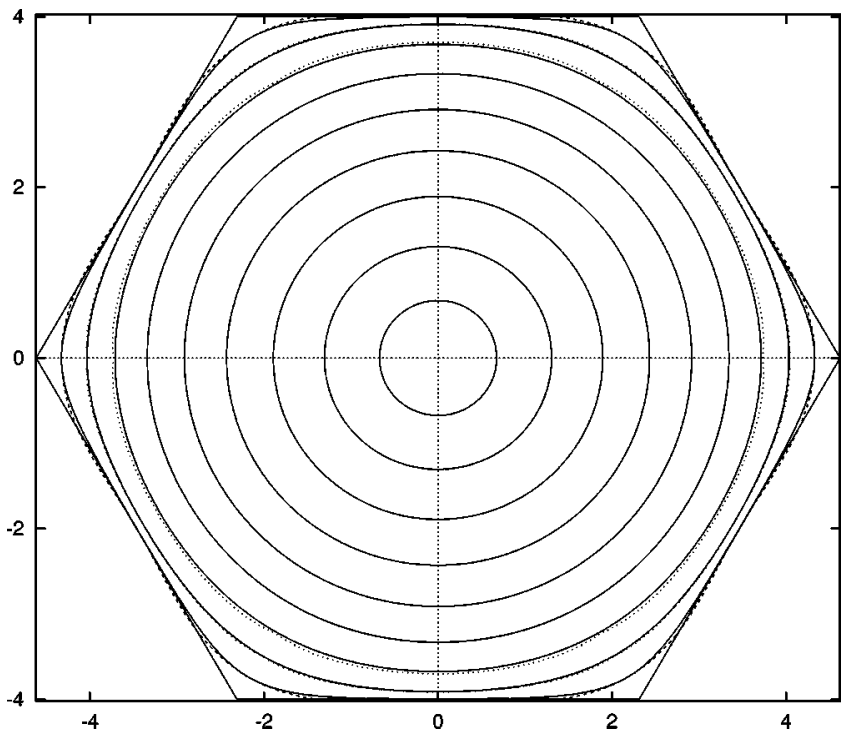

FIG. 1. The exact Wulff shapes (solid lines) from $T=0$ (outside hexagon) to $T=0.9 T_{c}$ (inner circle) in increments of $0.1 T_{c}$. Areas under these shapes correspond to $W / J^{2} \equiv \pi \sigma_{e f f}^{2} / J^{2}$ - see Fig. 2 - and determine the interfacial energy. Two elementary approximations-Eq. (29) (dashed) and Eqs. (26) and (31) (dotted line)-are shown for $T \leqslant 0.3 T_{c}$. Equation (29)— the hightemperature approximation-blends in with exact shapes at $T$ $\geqslant 0.2 T_{c}$; the low-temperature approximation [Eqs. (26) and (31)] blends in with solid lines at $T \leqslant 0.1 T_{c}$.

(8), which bracket $\sigma_{e f f}$. Anisotropy which is due to the difference between $\sigma_{1}$ and $\sigma_{2}$, indeed vanishes quickly near $T_{c}$, and we examine this in more detail below.

\section{A. Limit $T \rightarrow T_{c}$}

As $T$ becomes close to $T_{c}, A(L)$, approaches its minimum $A\left(L_{c}\right)$, with an asymptote

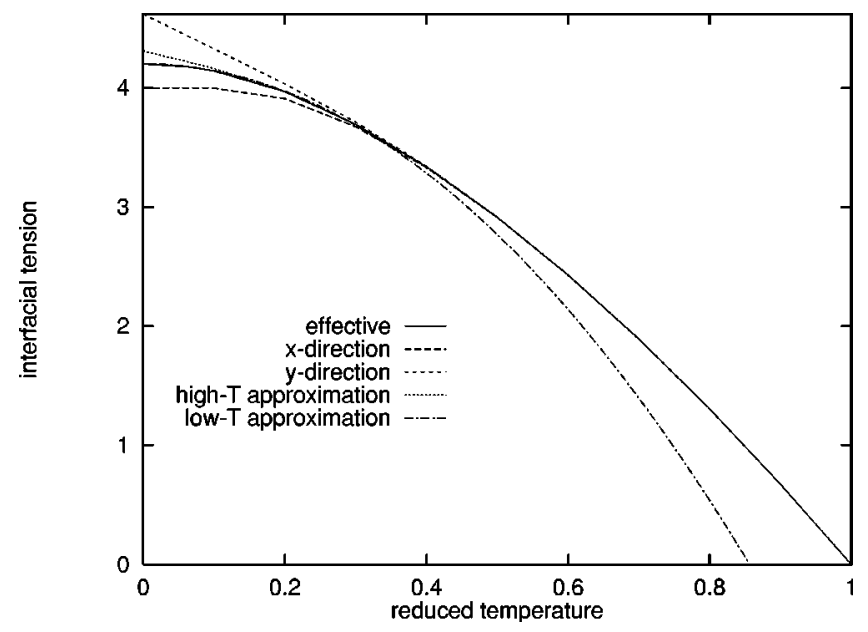

FIG. 2. Exact values for the reduced "effective" interfacial tension, $\sigma_{e f f} / J$ (solid line). The anisotropic tensions in the principal directions are indicated by short- and long-dashed lines, and approximations for $\sigma_{e f f}$ given by Eqs. (30) and (33) are shown by dotted and dash-dotted lines, respectively. 


$$
A-3 \sim 36\left(L-L_{c}\right)^{2} .
$$

This gives the interfacial tension-radii of smaller circles in Fig. 1-as

$$
\sigma_{c}(T) \sim 4 J \sqrt{3}\left(1-T / T_{c}\right)
$$

At lower temperatures we define a dimensionless "radius",

$$
\rho(\theta, T) \equiv r / T
$$

with

$$
\rho_{c}(T)=2 \sqrt{A / 3-1} \sim \sigma_{c}(T) / T_{c},
$$

determining the leading isotropic term near $T_{c}$.

Expanding the left-hand side of Eq. (4) to the sixth power in $\rho_{c}$ (the first term where the anisotropy appears), and solving the resulting equation iteratively, one obtains

$$
\begin{aligned}
\rho^{2}(\theta, T)= & \rho_{c}(T)^{2}-\frac{1}{16} \rho_{c}(T)^{4}+\frac{7}{9 \times 128} \rho_{c}(T)^{6} \\
& +\frac{1}{5760} \rho_{c}(T)^{6} \cos (6 \phi)
\end{aligned}
$$

Here, strictly speaking, an exact value of $\rho_{c}$ in Eq. (22) rather than its asymptote should be used to ensure the accuracy of higher-order terms in the isotropic part. An exceptionally minor anisotropy, even for $\rho_{c}$, of the order of unity should be noted. In principle, Eq. (23) can be used for evaluating $S$ shapes at intermediate temperatures (i.e., not only in the strict asymptotic limit), although a less rigorous but practically more convenient (and accurate) approximation will be constructed in Sec. V C.

\section{B. Limit $T \rightarrow 0$}

Let us introduce an auxiliary function

$$
m(\theta)=\max \{|\sin \theta|,|\sin (\theta+\pi / 3)|,|\sin (\theta-\pi / 3)|\},
$$

so that

$$
r_{0}(\theta)=4 J / m(\theta)
$$

describes the zero-temperature hexagon in Fig. 1 with an area

$$
W_{0}=32 \sqrt{3} J^{2}
$$

When considering the case of $T>0$, care should be taken since $r_{0}(\theta)$ is nonanalytic. The major modification comes from the disappearance of sharp corners-see Fig. 1-and in this section we will use the ideas of singular perturbations in order to evaluate the corresponding reduction in the area.

We look for a correction

$$
r(\theta)=r_{0}(\theta)(1+\delta(\theta) / 4 L) .
$$

From Eq. (4) and the low-temperature asymptote of $A(L)$, one has

$$
\delta(\theta)=-\ln \left(1+e^{-8|\theta| L / \sqrt{3}}\right), \quad|\theta| \ll 1 .
$$

Despite the restrictions on $\theta$, this expression is sufficient to evaluate the corrections to the area. Indeed, the main contribution to the integral in Eq. (17) comes from the region $\theta$ $=O(1 / L)$, which is well within the region of applicability of Eq. (27). Switching to a "stretched variable" $8 \theta L / \sqrt{3}$, and using the identity

$$
\int_{0}^{\infty} d z \ln \left(1+e^{-z}\right)=\pi^{2} / 12
$$

one obtains

$$
W_{0}-W(T) \sim \frac{2 \pi^{2} T^{2}}{\sqrt{3}} J^{2}=151.0776397 \cdots J^{2}\left(\frac{T}{T_{c}}\right)^{2} .
$$

Despite the exponential closeness of $r(\theta)$ to $r_{0}(\theta)$ in almost any direction, corrections to $W_{0}$ are quadratic in temperature, reflecting the "chop off" of sharp corners in Fig. 1. [In this context, note that corrections to $\sigma(\vec{n})$ in principal directions are, respectively, linear or exponentially small in $T$, with the resulting modification of the overall interfacial energy of a crystal having an intermediate quadratic dependence.] A similar quadratic correction, with a different proportionality constant derived numerically, was also observed for a square lattice, ${ }^{18}$ where an explicit integral expression of $W(T)$ through elliptic functions is available. ${ }^{10}$ In the present study the constant in Eq. (28) could be evaluated analytically, confirming, in a sense, the advantage of implicit parametric representations.

\section{Intermediate temperatures}

In the high-temperature region we introduce the approximation

$$
r(\theta, \quad T) \approx \frac{\sigma_{1}(T)+\sigma_{2}(T)}{2}+\frac{\sigma_{1}(T)-\sigma_{2}(T)}{2} \cos 6 \theta,
$$

with the anisotropic part suggested by the expansion near $T_{c}$. This expression is exact in the directions of extrema, $\theta$ $=l \pi / 6, l=0,1, \ldots, 11$, at any temperature. Otherwise, it correctly describes the location of the $S$ surface for temperatures as cold as $0.2 T_{c}$ - see Fig. 1. On the "experimental" level of accuracy the agreement is reasonable even at $0.1 T_{c}$; however, the low-temperature approximation, which will be described below, is more adequate and more accurate here.

In approximation (29) the anisotropic term does not contribute to the area, and $\sigma_{e f f}$ is given by

$$
\sigma_{e f f}(T) \approx \frac{\sigma_{1}(T)+\sigma_{2}(T)}{2}, \quad T \gtrsim 0.2 T_{c}
$$

with a relative error less than $10^{-3}$. The error rapidly decreases with the increase of temperature, as suggested by Fig. 2. At $0.5 T_{c}$, for example, Eq. (30) deviates from the exact value of $\sigma_{\text {eff }} \simeq 2.91280 \mathrm{~J}$ by less than one part in $10^{6}$. The Bessel-function approximation [Eq. (11)] can also be 
used for an evaluation of $\sigma_{e f f}$; however, Eq. (30) is slightly more accurate, and being an elementary function looks more attractive for applications.

At lower temperatures we construct an interpolating approximation for $\delta(\theta)$ in Eq. (26)

$$
\begin{aligned}
\delta(\theta) \approx & -\ln \left[(2 A)^{|\sin (\theta)| / m(\theta)-1}+(2 A)^{|\sin (\theta+\pi / 3)| / m(\theta)-1}\right. \\
& \left.+(2 A)^{|\sin (\theta-\pi / 3)| / m(\theta)-1}\right],
\end{aligned}
$$

with

$$
2 A \approx \exp (4 L) .
$$

Equation (31) coincides with Eq. (27) in the appropriate limits, but has no restrictions in $\theta$.

It can be used when plotting the low-temperature Wulff shapes for a triangular lattice (see Fig. 1). The approximation is very accurate up to $0.2 T_{c}$. Remarkably, even at $0.3 T_{c}$ one still obtain a reasonable, near circular shape (with minor dimples, the birthmarks of zero-temperature corners), although Eq. (29) works better here, completely blending in with the exact result in the scale of the figure. (28)

The low-temperature expression for $\sigma_{\text {eff }}$ follows from Eq.

$$
\begin{aligned}
\sigma_{e f f}(T) & \simeq J\left(\frac{32 \sqrt{3}}{\pi}\right)^{1 / 2}\left[1-\frac{\pi^{2}}{96}\left(\frac{T}{J}\right)^{2}\right] \\
& =J\left(\frac{32 \sqrt{3}}{\pi}\right)^{1 / 2}\left[1-1.362886 \cdots\left(\frac{T}{T_{c}}\right)^{2}\right],
\end{aligned}
$$

and is shown by dash-dotted lines in Fig. 2. The error in the region $T<0.2 T_{c}$ also does not exceed $10^{-3}$, and the equation becomes exact for $T \rightarrow 0$. Thus the two proposed approximations, [Eqs. (30) and (33)] cover the entire region of temperatures from zero to $T_{c}$.

\section{RELATION TO THE NUCLEATION PROBLEM}

Consider a small positive field $h$ and spins originally pointing in the "wrong" direction. The free-energy change associated with formation of a large domain of properly oriented spins with volume (area) $V$ is given by

$$
\Delta G(V)=2(V W)^{1 / 2}-2 h J V \kappa .
$$

Here $\kappa(T)$ is the equilibrium density of up-spins in a triangular lattice,

$$
\kappa=\frac{2}{\sqrt{3}} \chi(T),
$$

and $\chi(T)$ is the equilibrium magnetization ${ }^{29}$ (the values of $\chi$, however, are extremely close to unity anywhere away from $T_{c}$ ).

With the effective interfacial tension $\sigma_{\text {eff }}$ defined above, the critical volume is given by

$$
V_{*}=\frac{\pi \sigma_{e f f}^{2}}{4 h^{2} J^{2} \kappa^{2}} .
$$

This locates the maximum of $\Delta G(V)$, and the corresponding value

$$
G_{*} \equiv \Delta G\left(V_{*}\right)=\sigma_{e f f}\left(\pi V_{*}\right)^{1 / 2}
$$

determines the barrier to nucleation, as discussed by Gibbs.

Evaluation of the nucleation rate requires a specification of the spin-flip dynamics, which is not part of the present work. However, on this stage certain conclusions on applicability of some standard nucleation approaches can already be made, and this will be briefly discussed in Sec. VII.

\section{DISCUSSION}

In the present work exact equilibrium Wulff shapes were constructed for a triangular Ising lattice. The shapes bear strong qualitative similarities to the case of a honeycomb lattice, ${ }^{12}$ changing from a perfect hexagon at $T=0$ to a perfect circle near $T_{c}$. Quantitatively, however, the temperature dependence is different for each of these lattices.

Asymptotic analysis of exact expressions was performed near $T=0$ and $T=T_{c}$, and accurate elementary approximations were constructed for the entire temperature region. This part of the study can be potentially useful for applications.

For example, experimental studies of islands on a $\mathrm{Cu}(111)$ surface $^{5}$ fall into the low-temperature asymptotic region. There does not yet exist a uniform opinion on the values of $J$ for this system, but if one accepts $J \approx 0.16 \mathrm{eV}$ - one of the values used in the above paperone obtains $T_{c} \approx 0.58 \mathrm{eV}$. This is between 18 and 22 times larger than experimental temperatures used in Ref. 5. Even with uncertainties in $J$, there is little doubt that the ratio of $T / T_{c}$ is small, and the observed islands ${ }^{5}$ are indeed close to hexagons.

From Eqs. (25), (26), and (31) (also see Fig. 1) it follows that the only observable effect at small $T>0$ could be the erosion of sharp corners of the hexagon, again in qualitative agreement with observations in Ref. 5. Otherwise, the faces of a two-dimensional crystal remain practically flat or, more rigorously, exponentially close to flat. Correction to the interfacial energy is quadratic in temperature, and for such low $T$ the asymptotic expression [Eq. 33)] is expected to be very accurate. This can be helpful for further interpretation of experimental data.

The potential importance of discreteness effects ("finitesize" effects ${ }^{6}$ ) at low temperatures should be noted, however. Applicability of the aforementioned correction requires a large number of atoms lost from corners, which could be a nontrivial requirement even if $n$-the number of atoms in a two-dimensional crystal-is very large. One estimates the loss from each corner as $0.5 n\left(T / T_{c}\right)^{2}$; this falls in the interval between 4 and 14 atoms for $4000<n<9000$ reported in Ref. 5. Discreteness effects for such small numbers are possible, and the quadratic (in temperature) correction to interfacial energy, which is valid strictly for $n \rightarrow \infty$, should be treated with care.

If the islands are so small that discreteness changes the zero-temperature energy itself, "clusters" rather than Wulffshaped droplets should be considered. For a square Ising lattice this was recognized by Neves and Schonmann, ${ }^{13}$ and 
the role of such clusters in low-temperature nucleation received further attention in Refs. 30 and 17. There are no equivalent studies for hexagonal lattices.

The obtained high-temperature approximations for the Wullf shapes and energies can be relevant to the analysis of large-scale simulations of nucleation and growth of islands in Ising systems. Higher temperature is usually selected in such simulations in order to ensure detectable nucleation rates. The nucleus here is a practically circular droplet, with the interfacial tension accurately given by an elementary equation (30) [or by Eq. (11)]; input of the small anisotropy to the shape of a crystal can be estimated from Eq. (29) or (23).

Results obtained in Sec. VI for the free energy of formation of a nucleus can be used to specify parameters in several mainstream nucleation theories, after which they can be tested against simulation data. For example, one can now estimate the nucleation rate or cluster distributions in the classical nucleation theory ${ }^{19-21}$ when applied to a triangular lattice. Those estimations can be compared with data of Ref. 18 (simulations at $T \simeq 0.82 T_{c}$ ), and the comparison allows one to rule out the classical theory at this temperature since it predicts unrealistically small slopes for both the fielddependent nucleation rate and the size-dependent distributions. This could be an important conclusion, even if negative, due to an extraordinary status of that theory in understanding the nucleation phenomena. More recent approaches $^{31-34}$ seem to work more accurately here, simi- larly to the better studied case of a square lattice, ${ }^{15,16}$ but more simulation data are required for any definite conclusions.

\section{APPENDIX: HONEYCOMB LATTICE}

All of the above expressions which do not yet specify the temperature dependence but contain only the function $A(L)$ are valid for the honeycomb case as well, with $A(L)$ replaced by $A(K)$ from Eqs. (2) and (6). Specifying $A(K)$, for $T \rightarrow T_{c}$ one obtains:

$$
\sigma_{c}^{h o n}(T) \sim 4 J^{\prime}\left(1-T / T_{c}\right)
$$

(note a somewhat unexpected integer value of the slope). This asymptote determines $\rho_{c}$ in the high-temperature expansion [Eq. (23)]. The intermediate approximations for the Wulff shapes and energies, [Eqs. (29) and (30)] still can be used with appropriate values of $A(K)$ in Eqs. (7) and (8).

The low-temperature approximation for the Wulff shape is given by Eq. (31) with

$$
A(K) \approx e^{2 K} / 2
$$

From the analysis for the triangular case, switching from low- to high-temperature approximations (with errors in energies less then $10^{-3}$ ) should be done around $A=120$. This corresponds to $0.24 T_{c}$ in the honeycomb case, compared to $0.2 T_{c}$ in the case of a triangular lattice.
${ }^{1}$ G. Wulff, Z. Kryst Mineral. 34, 449 (1901).

${ }^{2}$ A.A. Chernov, Modern Crystallography III, Crystal Growth (Springer, Berlin, 1984).

${ }^{3}$ C. Rottman and M. Wortis, Phys. Rep. 103, 59 (1984).

${ }^{4}$ R.K.P. Zia, Statistical and Particle Physics-Common Problems and Techniques, edited by K. C. Bowler and A. J. McKane (SUSSP, Edinburgh, 1984), p. 247.

${ }^{5}$ G. Schulze Icking-Konert, M. Giesen, and H. Ibach, Phys. Rev. Lett. 83, 3880 (1999).

${ }^{6}$ M. Giesen, C. Steiner, and H. Ibach, Surf. Sci. (to be published).

${ }^{7}$ B. Berge, L. Faucheux, K. Schwab, and A. Libchaber, Nature (London) 350, 322 (1991).

${ }^{8}$ W.K. Burton, N. Cabrera, and F.G. Frank, Philos. Trans. R. Soc. London, Ser. A 243, 299 (1951).

${ }^{9}$ D.W. Hoffman and J.W. Cahn, Surf. Sci. 31, 369 (1972).

${ }^{10}$ R.K.P. Zia and J.E. Avron, Phys. Rev. B 25, 2042 (1982).

${ }^{11}$ J.E. Avron, H. van Beijeren, L.S. Schulman, and R.K.P. Zia, J. Phys. A 15, L81 (1982).

${ }^{12}$ R.K.P. Zia, J. Stat. Phys. 45, 801 (1986).

${ }^{13}$ E.J. Neves and R.H. Schonmann, Commun. Math. Phys. 137, 209 (1991).

${ }^{14}$ C.C. Günther, P.A. Rikvold, and M.A. Novotny, Phys. Rev. Lett. 71, 3898 (1993); Physica A 212, 194 (1994).

${ }^{15}$ P.A. Rikvold, H. Tomita, S. Miyashita, and S.W. Sides, Phys. Rev. E 49, 5080 (1994).

${ }^{16}$ R.A. Ramos, P.A. Rikvold, and M.A. Novotny, Phys. Rev. B 59, 9053 (1999).
${ }^{17}$ V.A. Shneidman, K.A. Jackson, and K.M. Beatty, J. Chem. Phys. 111, 6932 (1999).

${ }^{18}$ V.A. Shneidman, K.A. Jackson, and K.M. Beatty, Phys. Rev. B 59, 3579 (1999).

${ }^{19}$ M. Volmer and A. Weber, Z. Phys. Chem., Stoechiom. Verwandtschaftsl. 119, 227 (1926).

${ }^{20}$ R. Becker and W. Döring, Ann. Phys. (N.Y.) 24, 719 (1935).

${ }^{21}$ Ya.B. Zeldovich, Acta Physiochim. (URSS) 18, 1 (1943).

${ }^{22}$ V.A. Shneidman, Zh. Tekh. Fiz. 57, 131 (1987) [Sov. Phys. Tech. Phys. 32, 76 (1987)]; 58, 1102 (1988) [33, 1338 (1988)].

${ }^{23}$ L. Onsager, Phys. Rev. 65, 117 (1944).

${ }^{24}$ A.E. Ferdinand, Ph.D. thesis. University of London, 1967; P.G. Watson, J. Phys. C 1, 575 (1968); M.E. Fisher, J. Phys. Soc. Jpn. 26, 87 (1969); R.K.P. Zia, Phys. Lett. A 64, 345 (1978); E. Fradkin, B.A. Huberman, and S.H. Shenker, Phys. Rev. B 18, 4780 (1978).

${ }^{25}$ R.J. Baxter, Exactly Solved Models in Statistical Mechanics (Academic Press, New York, 1982).

${ }^{26}$ H.G. Vaidya, Phys. Lett. A 57, 1 (1976).

${ }^{27}$ I. Syozi, in Phase Transitions and Critical Phenomena, edited by C. Domb and M. S. Green (Academic Press, London, 1971), p. 270.

${ }^{28}$ G.H. Wannier, Rev. Mod. Phys. 17, 50 (1945).

${ }^{29}$ B.M. McCoy and T.T. Wu. The Two-Dimensional Ising Model (Harvard University Press, Cambridge, MA, 1973).

${ }^{30}$ M.A. Novotny, in Computer Simulation Studies in CondensedMatter Physics IX, edited by D.P. Landau, K.K. Mon, and H.-B. 
Schüttler, Springer Proceedings in Physics Vol. 82 (Springer, Berlin, 1997), p. 182.

${ }^{31}$ J.S. Langer, Ann. Phys. (N.Y.) 54, 258 (1969).

${ }^{32}$ J.S. Langer, Ann. Phys. (N.Y.) 65, 53 (1971).
${ }^{33}$ J.D. Gunton, M. San Miguel, and P.S. Sahni, in Phase Transitions and Critical Phenomena, edited by C. Domb and J.L. Lebowitz (Academic, New York, 1983), Vol. 8. p. 267.

${ }^{34}$ R.K.P. Zia and D.J. Wallace, Phys. Rev. B 31, 1624 (1985). 